\title{
Convergence Properties and Numerical Approximation of the Solution of the Mindlin Plate Bending Problem
}

\author{
By Roger Pierre
}

\begin{abstract}
We study the behavior of the solution of the Mindlin problem when the thickness becomes small, paying particular attention to the shear stress. We also propose a modification of a known scheme that allows the use of linear finite element approximation and we prove optimal error bounds.
\end{abstract}

1. Introduction. In plate theory many useful formulations are obtained by replacing, in the three-dimensional model of linear elasticity, the space of displacements and stresses by a well-chosen subspace in such a way as to obtain a two-dimensional problem which contains the thickness $t$ as a parameter. In [5], Destuynder analyzed this approach thoroughly and showed that some of the resulting models were plagued with boundary layer effect when the thickness is tending to zero. This effect is often linked to the "locking phenomenon" discussed in the engineering literature and is known to complicate the numerical determination of some of the variables.

In [3], Brezzi and Fortin studied one of these approximations, namely the Mindlin-Reissner model, for which they proposed a different formulation allowing efficient calculation of the critical unknowns. We come back to their decomposition procedure and show that it can be used in conjunction with singular perturbation to give a rather complete description of the limit process. We also prove that, under a slight modification, this method can be adapted to the use of linear finite elements for each of the variables, still being optimal and uniformly good as $t$ goes to zero.

The outline of the paper is as follows. We present the model in Section 2 and the Brezzi-Fortin decomposition in Section 3. In Section 4 we study an asymptotic expansion of the solution and prove a convergence result. Finally, in Section 5, we propose a simple discretization and get optimal and uniform error bounds.

2. Presentation of the Model. Let $\omega$ be an open bounded simply connected set of $\mathbf{R}^{2}$ with a $C^{\infty}$ boundary $\gamma$. For $t>0$, we denote by $\Omega_{t}$ the set $\Omega_{t}=$ $\omega \times[-t,+t]$. We suppose that $\Omega_{t}$ describes the undeformed configuration of a Saint-Venant Kirchhoff plate. If this plate is clamped along its vertical boundary $\gamma \times[-t,+t]$ and is acted upon by a vertical body force of density $\left(0,0, f_{3}\right)$, the

Received June 8, 1987.

1980 Mathematics Subject Classification (1985 Revision). Primary 65N30, 73K10, 73K25. 
general displacement problem can be written

$$
\text { Determine } \inf _{V^{t}} \int_{\Omega_{t}}\left\{\frac{\lambda}{2}(\operatorname{tr}(\varepsilon(\mathbf{v})))^{2}+\mu\left(\operatorname{tr}\left(\varepsilon^{2}(\mathbf{v})\right)\right)-\mathbf{f} \cdot \mathbf{v}\right\} d \mathbf{x},
$$

where $\varepsilon(\mathbf{v})$ is the strain tensor, $\lambda$ and $\mu$ the Lamé coefficients of the material and $V^{t}$ the space of admissible displacements. Replacing $V^{t}$ by

$$
V^{m, t}=\left\{\mathbf{v} \in V^{t} \mid \mathbf{v}=\left(-z \beta_{1}(x, y),-z \beta_{2}(x, y), w(x, y)\right), \beta_{1}, \beta_{2}, w \in H_{0}^{1}(\omega)\right\}
$$

we obtain a model which is very similar to Mindlin's model as presented in [1] but may also be looked upon as a particular case of Nagdhi's model of order one studied, for example, in [5]. For $\mathbf{v} \in V^{m, t}, \varepsilon(\mathbf{v})$ can be written in the block form

$$
\varepsilon(\mathbf{v})=\left[\begin{array}{cc}
-z \varepsilon(\boldsymbol{\beta}) & \frac{1}{2}(\nabla w-\boldsymbol{\beta}) \\
\frac{1}{2}(\nabla w-\boldsymbol{\beta})^{t} & 0
\end{array}\right]
$$

and, upon integrating with respect to the thickness in (1), we are led to the problem

$$
\text { Determine } \inf _{(\boldsymbol{\beta}, w)} \frac{t^{3}}{3} a(\boldsymbol{\beta}, \boldsymbol{\beta})+\frac{\mu t}{2} \int_{\omega}\|\nabla w-\boldsymbol{\beta}\|^{2} d \mathbf{x}-\int_{\Omega_{t}} f_{3} w d \mathbf{x},
$$

where $a(\boldsymbol{\beta}, \boldsymbol{\beta})$ is the two-dimensional deformation energy corresponding to

$$
a(\boldsymbol{\beta}, \boldsymbol{\eta})=\int_{\omega}\left\{2 \mu \boldsymbol{\varepsilon}(\boldsymbol{\beta})+\lambda \operatorname{tr}(\boldsymbol{\varepsilon}(\boldsymbol{\beta})) \mathbf{I}_{2}\right\}: \boldsymbol{\varepsilon}(\boldsymbol{\eta}) d \mathbf{x} .
$$

In [3] Brezzi and Fortin studied the behavior of the solutions of Mindlin's problem as the thickness $t$ goes to zero, in the particular case $f_{3}=t^{2} g(x, y) / 3$. This choice was motivated by the necessity of having solutions which were, at least, bounded in $t$. Integrating the last term with respect to $z$ and then dividing throughout by $2 t^{3} / 3$, we are finally led to the problem

$$
\text { Determine } \inf _{(\boldsymbol{\beta}, w)} \frac{1}{2} a(\boldsymbol{\beta}, \boldsymbol{\beta})+\frac{3 \mu}{4 t^{2}}|\nabla w-\boldsymbol{\beta}|_{0}^{2}-(g, w)
$$

(with the usual $L^{2}(\omega)$-norm and inner product). For the sake of simplicity, from now on we set

$$
\nu=\frac{3 \mu}{2} \text {. }
$$

Remark 1. In this form, Mindlin's problem is nothing but a penalty formulation of Kirchhoff's constrained problem

$$
\inf \left\{\frac{1}{2} a(\boldsymbol{\beta}, \boldsymbol{\beta})-(g, w) \mid \nabla w=\boldsymbol{\beta}\right\} .
$$

Such a formulation is well known to engineers who are using it in conjunction with reduced integration to solve the classical plate problem. (See [7].)

This version of Mindlin's model differs from the one given in [1] only in the values of the constant coefficients appearing in (4). Since the determination of these constants depends on technical a priori assumptions, and since their values do not modify the mathematical properties of the solution, we will no longer distinguish between the two approaches. 
3. A Mixed Formulation of the Problem. In [3] Brezzi and Fortin proposed a variational formulation of (4) based on an orthogonal decomposition of $\nabla w-\beta$. We would like to show that this decomposition is, in a certain sense, natural.

Let us denote the functional appearing in $(4)$ by $j(\boldsymbol{\beta}, w)$ and compute its Gateaux derivative at $(\beta, w)$ in the direction $(0, \xi)$. We get

$$
D_{(0, \xi)} j(\boldsymbol{\beta}, w)=\frac{\nu}{t^{2}}(\nabla w-\beta, \nabla \xi)-(g, \xi) .
$$

Hence, at the point $(\boldsymbol{\beta}, w)$ where the extremum is attained, we have

$$
\frac{\nu}{t^{2}} \operatorname{div}(\nabla w-\boldsymbol{\beta})=-g \text {. }
$$

Now, if $g \in L^{2}(\omega)$, we can write $g=-\Delta \phi$ with $\phi \in H_{0}^{1}(\omega) \cap H^{2}(\omega)$ and obtain

$$
\operatorname{div}\left(\frac{\nu}{t^{2}}(\nabla w-\beta)-\nabla \phi\right)=0
$$

from which we deduce that there exists a $p \in H^{1}(\omega) / \mathbb{R}$ such that

$$
\nabla w-\beta=\frac{t^{2}}{\nu}(\nabla \phi+\operatorname{rot} p)
$$

This reasoning indicates that the following well-known decomposition

$$
\left(L^{2}(\omega)\right)^{2}=\operatorname{grad}\left(H_{0}^{1}(\omega)\right) \oplus \operatorname{rot}\left(H^{1}(\omega) / \mathbf{R}\right)
$$

plays a fundamental role and leads us to the following theorem, the proof of which can be found in [3].

THEOREM A. Let $g$ be in $L^{2}(\omega)$ and $\phi \in H_{0}^{1}(\omega) \cap H^{2}(\omega)$ be the unique solution of the Dirichlet problem

$$
(\nabla \phi, \nabla \psi)=(g, \psi) \quad \forall \psi \in H_{0}^{1}(\omega) .
$$

Problem (4) has a unique solution $(\boldsymbol{\beta}, w)$ for which there exists a $p \in H^{1}(w) / \mathbf{R}$ such that (5) is true. Moreover, $(\boldsymbol{\beta}, w)$ is the solution of (4) if and only if $(\boldsymbol{\beta}, p, w)$ satisfies

$$
(\mathscr{P})\left\{\begin{array}{l}
a(\boldsymbol{\beta}, \boldsymbol{\eta})-(\operatorname{rot} p, \boldsymbol{\eta})=(\nabla \phi, \boldsymbol{\eta}) \quad \forall \boldsymbol{\eta} \in\left(H_{0}^{1}(\omega)\right)^{2} \\
-(\boldsymbol{\beta}, \operatorname{rot} q)=\frac{t^{2}}{\nu}(\operatorname{rot} p, \operatorname{rot} q) \quad \forall q \in H^{1}(\omega) / \mathbf{R}, \\
(\nabla w, \nabla \xi)=(\boldsymbol{\beta}, \nabla \xi)+\frac{t^{2}}{\nu}(\nabla \phi, \nabla \xi) \quad \forall \xi \in H_{0}^{1}(\omega) .
\end{array}\right.
$$

Remark 2. Because of its practical importance, we will discuss the behavior of the variable

$$
\Gamma_{t}=\frac{\nu}{t^{2}}(\nabla w-\beta)
$$

appearing in (5) and related to the shear stress.

The following a priori estimates, given in [3], will play a key role in the sequel.

THEOREM B. Let $\left(\boldsymbol{\beta}_{t}, p_{t}, w_{t}\right)$ be the solution of $(\mathscr{P})$. We have

$$
\left\|\boldsymbol{\beta}_{t}\right\|_{2}+\left\|w_{t}\right\|_{2}+\left|p_{t}\right|_{1}+t\left|p_{t}\right|_{2} \leq c|g|_{0}
$$

with $c$ independent of $t$. 
4. Solution Behavior for Small $t$. To study the behavior of the solution of $(\mathscr{P})$ as $t$ goes to zero, we first consider the limit problem:

$$
\left(\mathscr{P}_{0}\right)\left\{\begin{array}{l}
a\left(\boldsymbol{\beta}_{0}, \boldsymbol{\eta}\right)-\left(p_{0}, \operatorname{rot} \boldsymbol{\eta}\right)=(\nabla \phi, \boldsymbol{\eta}) \quad \forall \boldsymbol{\eta} \in\left(H_{0}^{1}(\omega)\right)^{2} \\
-\left(\operatorname{rot} \boldsymbol{\beta}_{0}, q\right)=0 \quad \forall q \in H^{1}(\omega) / \mathbb{R}, \\
\left(\nabla w_{0}, \nabla \xi\right)=\left(\boldsymbol{\beta}_{0}, \nabla \xi\right) \quad \forall \xi \in H_{0}^{1}(\omega) .
\end{array}\right.
$$

Up to a rotation of the variable $\boldsymbol{\eta}$, the first two equations give nothing but a Stokes problem, whence they possess a unique solution in $\left(H_{0}^{1}(\omega)\right)^{2} \times L^{2}(\omega) / \mathbb{R}$. Moreover, in view of known regularity results (see [6]),

$$
p_{0} \in H^{s} / \mathbb{R} \quad \text { whenever } g \in H^{s-2} \text {. }
$$

We observe now that, whereas $(\mathscr{P})_{2}$ implies that $\operatorname{rot} p_{t} \cdot \tau=0$ on $\gamma$, this relation is generally false for $t=0$. This is a boundary layer which affects the convergence properties of the solution. To illustrate this, we consider the following formal expansion:

$$
\left(\boldsymbol{\beta}_{t}, p_{t}, w_{t}\right)=\sum_{\nu=0}^{\infty} t^{2 \nu}\left(\boldsymbol{\beta}_{2 \nu}, p_{2 \nu}, w_{2 \nu}\right)
$$

Substituting the right-hand side of $(10)$ in $(\mathscr{P})$ and identifying the various coefficients of successive powers of $t$, we are led to an infinite sequence of problems:

$$
\left(\mathscr{P}_{j}\right) \begin{cases}a\left(\boldsymbol{\beta}_{2 j}, \boldsymbol{\eta}\right)-\left(\operatorname{rot} p_{2 j}, \boldsymbol{\eta}\right)=\delta_{j, 0}(\nabla \phi, \boldsymbol{\eta}) & \forall \boldsymbol{\eta} \in\left(H_{0}^{1}(\omega)\right)^{2}, \\ -\left(\boldsymbol{\beta}_{2 j}, \operatorname{rot} q\right)=\frac{1}{\nu}\left(\operatorname{rot} p_{2 j-2}, \operatorname{rot} q\right) & \forall q \in H^{1}(\omega) / \mathbb{R}, \\ \left(\nabla w_{2 j}, \nabla \xi\right)=\left(\boldsymbol{\beta}_{2 j}+\frac{\delta_{j, 1}}{\nu} \nabla \phi, \nabla \xi\right) & \forall \xi \in H_{0}^{1}(\omega),\end{cases}
$$

where we set $p_{-2}=0$ and use the standard Dirac delta notation. Let us suppose that $\left(\mathscr{P}_{1}\right)$ possesses a solution and integrate the second equation by parts. In view of the above remark, we get that $\boldsymbol{\beta}_{2} \cdot \tau \neq 0$ on $\gamma$. Thus, the boundary layer on the $p$ component of the solution is reflected on the successive terms of the approximation of $\boldsymbol{\beta}_{\boldsymbol{t}}$. This also makes life worse, since both the inf-sup condition

$$
\inf _{p \in L^{2} / \mathbf{R}}\left(\sup _{(\boldsymbol{\eta} \in V)} \frac{(p, \text { rot } \boldsymbol{\eta})}{\|\boldsymbol{\eta}\|_{V}\|p\|_{L^{2} / \mathbf{R}}}\right) \geq c_{0}>0
$$

and the ellipticity condition

$$
a(\boldsymbol{\eta}, \boldsymbol{\eta}) \geq c_{a}\|\boldsymbol{\eta}\|_{V}
$$

are valid only on $V=\left(H_{0}^{1}(\omega)\right)^{2}$. Hence, the existence of a solution for the corresponding problem is not guaranteed.

The next result helps us overcome this difficulty.

LEMMA 1. Let $H_{n}^{1}$ be defined by

$$
H_{n}^{1}=\left\{\boldsymbol{\beta} \in\left(H^{1}(\omega)\right)^{2} \mid \boldsymbol{\beta} \cdot \mathbf{n}=0 \text { on } \gamma\right\}
$$

and equipped with the $\left(H^{1}(\omega)\right)^{2}$-norm. If $\mathbf{f} \in\left(H^{1}(\omega)\right)^{2}$, the problem

$$
\left\{\begin{array}{c}
a(\boldsymbol{\beta}, \boldsymbol{\eta})-\langle\operatorname{rot} p, \boldsymbol{\eta}\rangle_{-1,0}=0 \quad \forall \boldsymbol{\eta} \in\left(H_{0}^{1}(\omega)\right)^{2} \\
-(\boldsymbol{\beta}, \operatorname{rot} q)=(\mathbf{f}, \operatorname{rot} q) \quad \forall q \in H^{1}(\omega) / \mathbb{R}
\end{array}\right.
$$


possesses a unique solution in $H_{n}^{1} \times L^{2} / \mathbf{R}$. Moreover, if $\mathrm{f} \in\left(H^{s}\right)^{2}, s \geq 1$, then

$$
\|\boldsymbol{\beta}\|_{s}+\|p\|_{H^{s-1} / \mathbf{R}} \leq C_{0}\|\mathbf{f}\|_{s} .
$$

Proof. We first prove unicity. Let $\left(\boldsymbol{\beta}_{1}, p_{1}\right),\left(\boldsymbol{\beta}_{2}, p_{2}\right)$ be two solutions in $H_{n}^{1} \times L^{2} / \mathbf{R}$. We have

$$
\left(\mathscr{P}^{*}\right)\left\{\begin{array}{l}
a\left(\boldsymbol{\beta}_{1}-\boldsymbol{\beta}_{2}, \boldsymbol{\eta}\right)-\left\langle\operatorname{rot}\left(p_{1}-p_{2}\right), \boldsymbol{\eta}\right\rangle_{-1,0}=0 \quad \forall \boldsymbol{\eta} \in H_{0}^{1}(\omega)^{2}, \\
-\left(\boldsymbol{\beta}_{1}-\boldsymbol{\beta}_{2}, \operatorname{rot} q\right)=0 \quad \forall q \in H^{1} / \boldsymbol{R} .
\end{array}\right.
$$

Integrating by parts, we deduce from the second equation that

$$
\operatorname{rot}\left(\boldsymbol{\beta}_{1}-\boldsymbol{\beta}_{2}\right)=0 \quad \text { in } \omega \quad \text { and } \quad\left(\boldsymbol{\beta}_{1}-\boldsymbol{\beta}_{2}\right) \cdot \boldsymbol{\tau}=0 \quad \text { on } \boldsymbol{\gamma} .
$$

Since both functions are in $H_{n}^{1}$, the second equality implies that $\boldsymbol{\beta}_{1}-\boldsymbol{\beta}_{2}$ is in $\left(H_{0}^{1}(\omega)\right)^{2}$. Setting $\boldsymbol{\eta}=\boldsymbol{\beta}_{1}-\boldsymbol{\beta}_{2}$ in $\left(\mathscr{P}^{*}\right)_{1}$ we infer from (15) and (12) that $\boldsymbol{\beta}_{1}=\boldsymbol{\beta}_{2}$. The fact that $p_{1}=p_{2}$ now follows directly from $\left(\mathscr{P}^{*}\right)_{1}$ and (11).

To prove existence, we first construct a function $\boldsymbol{\beta}_{\gamma} \in H_{n}^{1}$ such that $\operatorname{div}\left(\boldsymbol{\beta}_{\gamma}\right)=0$ and $\boldsymbol{\beta}_{\gamma} \cdot \boldsymbol{\tau}=-\mathbf{f} \cdot \boldsymbol{\tau}$ on $\gamma$. To this end, we solve the following biharmonic problem

$$
\begin{cases}\Delta^{2} \theta=0 & \text { in } \omega, \\ \theta=0 & \text { on } \gamma, \\ \nabla \theta \cdot \mathbf{n}=\mathbf{f} \cdot \boldsymbol{\tau} & \text { on } \gamma .\end{cases}
$$

If $\mathbf{f} \in\left(H^{s}(\omega)\right)^{2}$, then $\mathbf{f} \cdot \tau \in H^{s-1 / 2}(\gamma)$ and the problem possesses a solution in $H^{s+1}(\omega)$ satisfying

$$
\|\theta\|_{s+1, \omega} \leq C\|\mathbf{f} \cdot \tau\|_{s-1 / 2, \gamma} \leq C_{1}\|\mathbf{f}\|_{s, \omega} .
$$

Setting $\boldsymbol{\beta}_{\gamma}=\operatorname{rot} \theta$, we get the desired result and the estimate

$$
\left\|\boldsymbol{\beta}_{\gamma}\right\|_{s} \leq C_{1}\|\mathbf{f}\|_{s} \text {. }
$$

Next, we set $\boldsymbol{F}(\boldsymbol{\eta})=-a\left(\boldsymbol{\beta}_{\gamma}, \boldsymbol{\eta}\right)$. This is a continuous linear functional on $\left(H_{0}^{1}(\omega)\right)^{2}$, hence there exists an $\mathbf{f}_{1} \in\left(H^{-1}\right)^{2}$ such that

$$
\left\langle\mathbf{f}_{1}, \boldsymbol{\eta}\right\rangle_{-1,0}=-a\left(\boldsymbol{\beta}_{\gamma}, \boldsymbol{\eta}\right) .
$$

In particular, for $s \geq 2$, one can show, using (3) and the fact that $\operatorname{div}\left(\boldsymbol{\beta}_{\gamma}\right)=0$, that $\mathbf{f}_{1}=\mu \Delta \boldsymbol{\beta}_{\gamma}$. In any case, $\mathbf{f}_{1}$ will be in $\left(H^{s-2}\right)^{2}$ when $\mathbf{f} \in\left(H^{s}\right)^{2}$ and will satisfy

$$
\left\|\mathbf{f}_{1}\right\|_{s-2} \leq\|\mathbf{f}\|_{s} .
$$

Similarly, setting $f_{2}=\operatorname{rot}\left(\mathbf{f}+\boldsymbol{\beta}_{\gamma}\right)$, we have that $f_{2}$ is in $H^{s-1}$ when $\mathbf{f} \in\left(H^{s}\right)^{2}$ and satisfies

$$
\left\|f_{2}\right\|_{s-1} \leq C_{2}\|\mathbf{f}\|_{s}
$$

Now we consider the problem: Find $\left(\boldsymbol{\beta}_{\omega}, p\right) \in\left(H_{0}^{1}(\omega)\right)^{2} \times L^{2} / \mathbf{R}$ such that

$$
\left(\mathscr{P}^{\prime}\right)\left\{\begin{array}{l}
a\left(\boldsymbol{\beta}_{\omega}, \boldsymbol{\eta}\right)-(p, \operatorname{rot} \boldsymbol{\eta})=\left\langle\mathbf{f}_{1}, \boldsymbol{\eta}\right\rangle_{-1,0} \quad \forall \boldsymbol{\eta} \in\left(H_{0}^{1}(\omega)\right)^{2}, \\
-\left(\operatorname{rot} \boldsymbol{\beta}_{\omega}, q\right)=\left(f_{2}, q\right) \quad \forall q \in L^{2} / \mathbf{R} .
\end{array}\right.
$$

As mentioned earlier, this problem is nothing but a Stokes problem and thus possesses a unique solution for which the following estimate is valid when $f \in\left(H^{s}\right)^{2}$,

$$
\left\|\boldsymbol{\beta}_{\omega}\right\|_{s}+\|p\|_{H^{s-1} / \mathbf{R}} \leq C_{3}\|\mathbf{f}\|_{s}
$$


To conclude, set $(\boldsymbol{\beta}, p)=\left(\boldsymbol{\beta}_{\gamma}+\boldsymbol{\beta}_{\omega}, p\right) \in H_{n}^{1} \times\left(L^{2} / \mathbf{R}\right)$. From $\left(\mathscr{P}^{\prime}\right)_{1}$ we deduce that

$$
a(\boldsymbol{\beta}, \boldsymbol{\eta})-\langle\operatorname{rot} p, \boldsymbol{\eta}\rangle_{-1,0}=0 \quad \forall \boldsymbol{\eta} \in\left(H_{0}^{1}(\omega)\right)^{2} .
$$

Similarly, if in $\left(\mathscr{P}^{\prime}\right)_{2}$ we restrict the test functions to $H^{1} / \mathbb{R}$ and if we integrate both sides by parts, we get, using $\mathbf{f} \cdot \boldsymbol{\tau}+\boldsymbol{\beta}_{\boldsymbol{\gamma}} \cdot \boldsymbol{\tau}=0$ on $\gamma$, that

$$
-(\boldsymbol{\beta}, \operatorname{rot} q)=(\mathbf{f}, \operatorname{rot} q) \quad \forall q \in\left(H^{1} / \mathbf{R}\right) .
$$

This shows that $(\beta, p)$ is the desired solution while the estimate (14) is a direct consequence of (16) and (17). This completes the proof.

Coming back to the problems under consideration, we have

THEOREM 1. Let $k \geq 0, g \in H^{k}(\omega)$ be given and set

$$
\nu_{k}=\left[\frac{k}{2}\right]+1 \text {. }
$$

For each $0 \leq j \leq \nu_{k}$ problem $\left(\mathscr{P}_{j}\right)$ possesses a unique solution $\left(\boldsymbol{\beta}_{2 j}, p_{2 j}, w_{2 j}\right)$ in

$$
\left(H_{n}^{1} \cap\left(H^{k+3-2 j}\right)^{2}\right) \times\left(H^{k+2-2 j} / \mathbf{R}\right) \times\left(H_{0}^{1} \cap H^{k+4-2 j}\right),
$$

for which

$$
\left\|\boldsymbol{\beta}_{2 j}\right\|_{k+3-2 j}+\left\|p_{2 j}\right\|_{H^{k+2-2 j} / \mathbf{R}}+\left\|w_{2 j}\right\|_{k+4-2 j} \leq C\|g\|_{k} .
$$

Proof. For a fixed $k$, the proof proceeds by induction on $j$. We already analyzed the case $j=0$, for which $p_{-2}=0$ and the estimate (18) is a direct consequence of classical regularity results.

Now suppose that the result is true for a fixed $j \leq k / 2$. In that case, $j+1 \leq \nu_{k}$, and $\left(\mathscr{P}_{j+1}\right)_{1},\left(\mathscr{P}_{j+1}\right)_{2}$ is a problem of the form (13) with

$$
\mathbf{f}=\frac{1}{\nu} \operatorname{rot} p_{2 j} \in\left(H^{k+1-2 j}\right)^{2} \subset\left(H^{1}\right)^{2}
$$

whence it has a solution $\left(\boldsymbol{\beta}_{2 j+2}, p_{2 j+2}\right)$ in $\left(H_{n}^{1} \cap H^{k+1-2 j}\right) \times\left(H^{k-2 j} / \mathbf{R}\right)$. The existence and regularity of $w_{2 j+2}$ as solution of $\left(\mathscr{P}_{j+1}\right)_{3}$ is immediate.

Finally, set $s=k+1-2 j$ in (14). We infer from the usual estimate of the solution of the Dirichlet problem that

$$
\begin{aligned}
\left\|\boldsymbol{\beta}_{2 j+2}\right\|_{k+3-2(j+1)} & +\left\|p_{2 j+2}\right\|_{H^{k+2-2(j+1)} / \mathbf{R}} \\
& +\left\|w_{2 j+2}\right\|_{k+4-2(j+1)} \leq C\left\|\operatorname{rot} p_{2 j}\right\|_{k+1-2 j} .
\end{aligned}
$$

Thus (18) follows by induction. This completes the proof.

We conclude this section by giving an estimate for the error between $\left(\beta_{t}, p_{t}, w_{t}\right)$ and $\left(\beta_{0}, p_{0}, w_{0}\right)$ in the appropriate norm. For this we need the following result due to Destuynder [5, pp. 208-209].

LEMMA 2. If $\hat{p}$ is in $H^{2}(\omega) / \mathbf{R}$, there exists $a \theta \in\left(H^{1}(\omega)\right)^{2}$ such that

$$
\left\{\begin{array}{l}
\boldsymbol{\theta} \cdot \mathbf{n}=0, \\
\boldsymbol{\theta} \cdot \boldsymbol{\tau}=-\operatorname{rot} \hat{p} \cdot \tau, \\
|\boldsymbol{\theta}|_{0}+t\|\boldsymbol{\theta}\|_{1} \leq C t^{1 / 2},
\end{array}\right.
$$

where the constant $C$ is independent of $t$. 
This leads us to

THEOREM 2. Let $g \in L^{2}(\omega)$; then we have

$$
\left\|\boldsymbol{\beta}_{t}-\boldsymbol{\beta}_{0}\right\|_{1}+t\left|p_{t}-p_{0}\right|_{1}+\left\|w_{t}-w_{0}\right\|_{2} \leq C t^{3 / 2} .
$$

Proof. Let us first subtract $\left(\mathscr{P}_{0}\right)$ from $(\mathscr{P})$ term by term. We get

$$
\left(\mathscr{P}_{t}^{*}\right)\left\{\begin{array}{l}
a\left(\boldsymbol{\beta}_{t}-\boldsymbol{\beta}_{0}, \boldsymbol{\eta}\right)-\left(\operatorname{rot}\left(p_{t}-p_{0}\right), \boldsymbol{\eta}\right)=0 \quad \forall \boldsymbol{\eta} \in\left(H_{0}^{1}(\omega)\right)^{2}, \\
-\left(\boldsymbol{\beta}_{t}-\boldsymbol{\beta}_{0}, \operatorname{rot} q\right)=\frac{t^{2}}{\nu}\left(\operatorname{rot} p_{t}, \operatorname{rot} q\right) \quad \forall q \in H^{1}(\omega) / \mathbb{R}, \\
\left(\nabla\left(w_{t}-w_{0}\right), \nabla \xi\right)=\left(\left(\boldsymbol{\beta}_{t}-\boldsymbol{\beta}_{0}\right)+\frac{t^{2}}{\nu} \nabla \phi, \nabla \xi\right) \quad \forall \xi \in H_{0}^{1}(\omega) .
\end{array}\right.
$$

In view of the inf-sup condition (11), we deduce from $\left(\mathscr{P}_{t}^{*}\right)_{1}$ that

$$
\left\|p_{t}-p_{0}\right\|_{L^{2} / \mathbf{R}} \leq C\left\|\boldsymbol{\beta}_{t}-\boldsymbol{\beta}_{0}\right\|_{1} \text {. }
$$

Let us remark that, since $g \in L^{2}, p_{0} \in H^{2} / \mathbf{R}$. Thus, we may set $\hat{p}=p_{0}$ in Lemma 2. Corresponding to the $\theta$ thus obtained, we denote by $\chi$ the unique solution of the Neumann problem

$$
\frac{1}{\nu}(\operatorname{rot} \chi, \operatorname{rot} q)=(\boldsymbol{\theta}, \operatorname{rot} q) \quad \forall q \in H^{1} / \mathbb{R} .
$$

The following is immediate:

$$
\left\{\begin{array}{l}
|\chi|_{1} \leq C|\boldsymbol{\theta}|_{0} \leq C t^{1 / 2} \\
|\Delta \chi|_{0}=\nu|\operatorname{rot}(\boldsymbol{\theta})|_{0} \leq C t^{-1 / 2} \\
\operatorname{rot}\left(\chi+p_{0}\right) \cdot \tau=0
\end{array}\right.
$$

We set $\boldsymbol{\eta}=\boldsymbol{\beta}_{t}-\boldsymbol{\beta}_{0}, q=p_{t}-p_{0}$ and subtract the first two equations of $\left(\mathscr{P}_{t}^{*}\right)$. This leads to

$$
a\left(\boldsymbol{\beta}_{t}-\boldsymbol{\beta}_{0}, \boldsymbol{\beta}_{t}-\boldsymbol{\beta}_{0}\right)=-\frac{t^{2}}{\nu}\left(\operatorname{rot} p_{t}, \operatorname{rot}\left(p_{t}-p_{0}\right)\right)
$$

which we write as

$$
\begin{aligned}
a\left(\boldsymbol{\beta}_{t}-\boldsymbol{\beta}_{0}, \boldsymbol{\beta}_{t}\right. & \left.-\boldsymbol{\beta}_{0}\right)+\frac{t^{2}}{\nu}\left|p_{t}-p_{0}-\chi\right|_{1}^{2} \\
& =-\frac{t^{2}}{\nu}\left(\operatorname{rot}\left(p_{0}+\chi\right), \operatorname{rot}\left(p_{t}-p_{0}\right)\right)-\frac{t^{2}}{\nu}\left(\operatorname{rot}\left(p_{t}-p_{0}-\chi\right), \operatorname{rot} \chi\right) .
\end{aligned}
$$

Integrating the first term on the right by parts and using (21), we get after estimation

$$
\begin{aligned}
c_{a} \| \boldsymbol{\beta}_{t} & -\boldsymbol{\beta}_{0} \|_{1}^{2}+\frac{t^{2}}{\nu}\left|p_{t}-p_{0}-\chi\right|_{1}^{2} \\
& \leq \frac{t^{2}}{\nu}\left|\Delta\left(\chi+p_{0}\right)\right|_{0}\left\|p_{t}-p_{0}\right\|_{L^{2} / \mathbf{R}}+\frac{t^{2}}{\nu}\left|p_{t}-p_{0}-\chi\right|_{1}|\chi|_{1} .
\end{aligned}
$$

Using (20) and (21), we obtain

$$
\left\|\boldsymbol{\beta}_{t}-\boldsymbol{\beta}_{0}\right\|_{1}^{2}+t^{2}\left|p_{t}-p_{0}-\chi\right|_{1}^{2} \leq C\left[t^{4}\left(|\Delta \chi|_{0}+\left|\Delta p_{0}\right|_{0}\right)^{2}+t^{2}|\chi|_{1}^{2}\right] \leq C t^{3} .
$$

Thus, using (21) again and the triangle inequality, we finally have

$$
\left\|\boldsymbol{\beta}_{t}-\boldsymbol{\beta}_{0}\right\|_{1}+t\left|p_{t}-p_{0}\right|_{1} \leq C t^{3 / 2} \text {. }
$$


To conclude, we observe that $w_{t}-w_{0}$ is the solution of the homogeneous Dirichlet problem

$$
\Delta w=\nabla \cdot\left(\boldsymbol{\beta}_{t}-\boldsymbol{\beta}_{0}\right)-\frac{t^{2}}{\nu} g
$$

Since the right-hand side is in $L^{2}$, we deduce from (22) that

$$
\left|w_{t}-w_{0}\right|_{2} \leq C\left(\left\|\boldsymbol{\beta}_{1}-\boldsymbol{\beta}_{0}\right\|_{1}+t^{2}|g|_{0}\right) \leq C t^{3 / 2} .
$$

This completes the proof.

Remark 3. The main interest of this result lies in the fact that it gives $L^{2}$ convergence for the variable $\Gamma_{t}$, as opposed to the $H^{-1}$ (div)-convergence obtained in [3]. As to the rate of convergence, we conjecture that it is optimal for $p_{t}$ but not for the other two variables.

A similar approach would lead to estimates for the error between the solution and the partial sums in (10) (see [5] for many examples of the techniques involved).

5. Discretization and Error Bounds. We now suppose that $\omega$ is polygonal and remark that Theorem B is still valid in that case. Although problem $(\mathscr{P})_{1},(\mathscr{P})_{2}$ is a standard elliptic problem, some care must be taken in the discretization because the ellipticity constant goes to zero with $t$. In order to be able to use linear $C^{0}$ finite elements, in the discrete problem we add to the troublesome term a mesh-dependent one of the same form but containing an adjustable parameter.

To be more precise, let $\left\{\mathscr{T}_{h}\right\}_{h}$ be a sequence of regular triangulations of $\omega$. Set

$$
\begin{array}{r}
P_{h}=\left\{\phi\left|\phi \in C^{0}(\bar{\omega}), \phi\right|_{K} \in P_{1}, \forall K \in \mathscr{T}_{h}\right\}, \\
W_{h}=P_{h} \cap H_{0}^{1}(\omega), \quad V_{h}=\left(W_{h}\right)^{2} .
\end{array}
$$

Let $\phi_{h}$ be the solution of the discrete Dirichlet problem

$$
\left(\nabla \phi_{h}, \nabla \psi_{h}\right)=\left(g, \psi_{h}\right) \quad \forall \psi_{h} \in W_{h}
$$

For a given $\alpha>0$, we consider the following discretized version of problem $(\mathscr{P})$ : Find $\left(\beta_{h}, p_{h}, w_{h}\right) \in V_{h} \times P_{h} \times W_{h}$ such that

$$
\left(\mathscr{P}_{h}\right)\left\{\begin{array}{l}
a\left(\boldsymbol{\beta}_{h}, \boldsymbol{\eta}_{h}\right)-\left(\operatorname{rot} p_{h}, \boldsymbol{\eta}_{h}\right)=\left(\nabla \phi_{h}, \boldsymbol{\eta}_{h}\right) \quad \forall \boldsymbol{\eta}_{h} \in V_{h}, \\
-\left(\boldsymbol{\beta}_{h}, \operatorname{rot} q_{h}\right)=\frac{1}{\nu}\left(t^{2}+\alpha^{2} h^{2}\right)\left(\operatorname{rot} p_{h}, \operatorname{rot} q_{h}\right) \quad \forall q_{h} \in P_{h}, \\
\left(\nabla w_{h}, \nabla \xi_{h}\right)=\left(\boldsymbol{\beta}_{h}+\frac{t^{2}}{\nu} \nabla \phi_{h}, \nabla \xi_{h}\right) \quad \forall \xi_{h} \in W_{h} .
\end{array}\right.
$$

We prove the following

THEOREM 3. If $g \in L^{2}(\omega)$, problem $\left(\mathscr{P}_{h}\right)$ has a unique solution such that, if $\left(\boldsymbol{\beta}_{t}, p_{t}, w_{t}\right)$ is the solution of $(\mathscr{P})$, we have

$$
\left\|\boldsymbol{\beta}_{t}-\boldsymbol{\beta}_{h}\right\|_{1}+(t+\alpha h)\left|p_{t}-p_{h}\right|_{1}+\left\|w_{t}-w_{h}\right\|_{1} \leq c h\|g\|_{0},
$$

where $c$ is independent of $h$ and $t$.

Proof. Given $\boldsymbol{\beta}_{h}$, the existence of $w_{h}$ causes no problem, so we concentrate on the first two equations. For this, we define a norm on $V_{h} \times P_{h}$ by

$$
\left\|\left(\boldsymbol{\beta}_{h}, p_{h}\right)\right\|_{t}^{2}=\left\|\boldsymbol{\beta}_{h}\right\|_{1}^{2}+t^{2}\left|p_{h}\right|_{1}^{2}
$$


a bilinear form $A_{h}$ on $\left(V_{h} \times P_{h}\right)^{2}$ by

$$
\begin{aligned}
A_{h}\left(\left(\boldsymbol{\beta}_{h}, p_{h}\right),\left(\boldsymbol{\eta}_{h}, q_{h}\right)\right)= & a\left(\boldsymbol{\beta}_{h}, \boldsymbol{\eta}_{h}\right)-\left(\operatorname{rot} p_{h}, \boldsymbol{\eta}_{h}\right)+\left(\boldsymbol{\beta}_{h}, \operatorname{rot} q_{h}\right) \\
& +\frac{1}{\nu}\left(t^{2}+\alpha^{2} h^{2}\right)\left(\operatorname{rot} p_{h}, \operatorname{rot} q_{h}\right)
\end{aligned}
$$

and a linear form $F_{h}$ on $V_{h} \times P_{h}$ by

$$
F_{h}\left(\boldsymbol{\eta}_{h}, q_{h}\right)=\left(\nabla \phi_{h}, \eta_{h}\right)
$$

If we consider the problem

$$
A_{h}\left(\left(\boldsymbol{\beta}_{h}, p_{h}\right),\left(\boldsymbol{\eta}_{h}, q_{h}\right)\right)=F_{h}\left(\boldsymbol{\eta}_{h}, q_{h}\right) \quad \forall\left(\boldsymbol{\eta}_{h}, q_{h}\right) \in V_{h} \times P_{h},
$$

it follows from the inequality

$$
A_{h}\left(\left(\boldsymbol{\beta}_{h}, p_{h}\right),\left(\boldsymbol{\beta}_{h}, p_{h}\right)\right) \geq c_{a}\left\|\boldsymbol{\beta}_{h}\right\|_{1}^{2}+\frac{1}{\nu}\left(t^{2}+\alpha^{2} h^{2}\right)\left|p_{h}\right|_{1}^{2}>c_{t}\left\|\left(\boldsymbol{\beta}_{h}, p_{h}\right)\right\|_{t}^{2}
$$

that the problem has a unique solution in $V_{h} \times P_{h}$. Since this problem is obviously equivalent to $\left(\mathscr{P}_{h}\right)_{1},\left(\mathscr{P}_{h}\right)_{2}$, the same is true for $\left(\mathscr{P}_{h}\right)$.

To get an error estimate for $\left(\boldsymbol{\beta}_{t}, p_{t}\right)$, we first replace in $(\mathscr{P}) \boldsymbol{\eta}$ by $\eta_{h}, q$ by $q_{h}$ and then subtract $\left(\mathscr{P}_{h}\right)_{1}$ from $(\mathscr{P})_{1}$ and $\left(\mathscr{P}_{h}\right)_{2}$ from $(\mathscr{P})_{2}$ to get

$$
\begin{gathered}
a\left(\boldsymbol{\beta}_{t}-\boldsymbol{\beta}_{h}, \boldsymbol{\eta}_{h}\right)-\left(\operatorname{rot}\left(p_{t}-p_{h}\right), \boldsymbol{\eta}_{h}\right)=\left(\nabla\left(\phi-\phi_{h}\right), \boldsymbol{\eta}_{h}\right), \\
-\left(\boldsymbol{\beta}_{t}-\boldsymbol{\beta}_{h}, \operatorname{rot} q_{h}\right)-\frac{1}{\nu}\left(t^{2}+\alpha^{2} h^{2}\right)\left(\operatorname{rot}\left(p_{t}-p_{h}\right), \operatorname{rot} q_{h}\right) \\
=-\frac{1}{\nu} \alpha^{2} h^{2}\left(\operatorname{rot} p_{t}, \operatorname{rot} q_{h}\right) .
\end{gathered}
$$

Let $\left(\hat{\boldsymbol{\beta}}_{h}, \hat{p}_{h}\right) \in V_{h} \times P_{h}$ be arbitrary; the above equations can be rewritten as

$$
\begin{aligned}
& a\left(\hat{\boldsymbol{\beta}}_{h}-\right.\left.\boldsymbol{\beta}_{h}, \boldsymbol{\eta}_{h}\right)-\left(\operatorname{rot}\left(\hat{p}_{h}-p_{h}\right), \boldsymbol{\eta}_{h}\right) \\
&=\left(\nabla\left(\phi-\phi_{h}\right), \boldsymbol{\eta}_{h}\right)+a\left(\hat{\boldsymbol{\beta}}_{h}-\boldsymbol{\beta}_{t}, \boldsymbol{\eta}_{h}\right)-\left(\operatorname{rot}\left(\hat{p}_{h}-p_{t}\right), \boldsymbol{\eta}_{h}\right), \\
&-\left(\hat{\boldsymbol{\beta}}_{h}-\boldsymbol{\beta}_{h}, \operatorname{rot} q_{h}\right)-\frac{1}{\nu}\left(t^{2}+\alpha^{2} h^{2}\right)\left(\operatorname{rot}\left(\hat{p}_{h}-p_{h}\right), \operatorname{rot} q_{h}\right) \\
&=-\left(\hat{\boldsymbol{\beta}}_{h}-\boldsymbol{\beta}_{t}, \operatorname{rot} q_{h}\right)-\frac{1}{\nu}\left(t^{2}+\alpha^{2} h^{2}\right)\left(\operatorname{rot}\left(\hat{p}_{h}-p_{t}\right), \operatorname{rot} q_{h}\right) \\
& \quad-\frac{1}{\nu} \alpha^{2} h^{2}\left(\operatorname{rot} p_{t}, \operatorname{rot} q_{h}\right) .
\end{aligned}
$$

From now on, we select an arbitrary but fixed representative of the equivalence class of $p_{t}$ in $H^{1} / \mathbb{R}$, which we still denote by $p_{t}$. In the above equations we then set $\boldsymbol{\eta}_{h}=\hat{\boldsymbol{\beta}}_{h}-\boldsymbol{\beta}_{h}, q_{h}=\hat{p}_{h}-p_{h}$, subtract side by side, integrate the third term on the right by parts and estimate. We get

$$
\begin{aligned}
c_{a} \| \hat{\boldsymbol{\beta}}_{h}- & \boldsymbol{\beta}_{h} \|_{1}^{2}+\frac{1}{\nu}\left(t^{2}+\alpha^{2} h^{2}\right)\left|\hat{p}_{h}-p_{h}\right|_{1}^{2} \\
\leq & \left\|\phi_{h}-\phi\right\|_{1}\left\|\hat{\boldsymbol{\beta}}_{h}-\boldsymbol{\beta}_{h}\right\|_{1}+\left(\|a\|\left\|\boldsymbol{\beta}_{t}-\hat{\boldsymbol{\beta}}_{h}\right\|_{1}+\left|p_{t}-\hat{p}_{h}\right|_{0}\right)\left\|\hat{\boldsymbol{\beta}}_{h}-\boldsymbol{\beta}_{h}\right\|_{1} \\
& +\left|\hat{\boldsymbol{\beta}}_{h}-\boldsymbol{\beta}_{t}\right|_{0}\left|\hat{p}_{h}-p_{h}\right|_{1}+\frac{1}{\nu}\left(t^{2}+\alpha^{2} h^{2}\right)\left|\hat{p}_{h}-p_{t}\right|_{1}\left|\hat{p}_{h}-p_{h}\right|_{1} \\
& +\frac{1}{\nu} \alpha^{2} h^{2}\left|p_{t}\right|_{1}\left|\hat{p}_{h}-p_{h}\right|_{1} .
\end{aligned}
$$

In estimating the right-hand side, some care must be taken with the fourth term, since $\left|p_{t}\right|_{2}$ might not be bounded in $t$. Because of that, we split it and consider the 
$t^{2}$ and $h^{2}$ factors separately. Applying the arithmetic-geometric inequality to each term, we are led to

$$
\begin{aligned}
c_{a} \| \hat{\boldsymbol{\beta}}_{h}- & \boldsymbol{\beta}_{h} \|_{1}^{2}+\frac{1}{\nu}\left(t^{2}+\alpha^{2} h^{2}\right)\left|\hat{p}_{h}-p_{h}\right|_{1}^{2} \\
\leq & \frac{3}{c_{a}}\left\|\phi_{h}-\phi\right\|_{1}^{2}+\frac{3\|a\|^{2}}{c_{a}}\left\|\boldsymbol{\beta}_{t}-\hat{\boldsymbol{\beta}}_{h}\right\|_{1}^{2} \\
& +\frac{3}{c_{a}}\left|p_{t}-\hat{p}_{h}\right|_{0}^{2}+\frac{3}{\alpha^{2} h^{2}}\left|\hat{\boldsymbol{\beta}}_{h}-\boldsymbol{\beta}_{t}\right|_{0}^{2}+\frac{t^{2}}{\nu}\left|\hat{p}_{h}-p_{t}\right|_{1}^{2} \\
& +\frac{3 \alpha^{2}}{\nu} h^{2}\left|\hat{p}_{h}-p_{t}\right|_{1}^{2}+\frac{3 \alpha^{2}}{\nu} h^{2}\left|p_{t}\right|_{1}^{2} .
\end{aligned}
$$

In view of the definition of $\phi_{h}$, we have

$$
\left\|\phi-\phi_{h}\right\|_{1} \leq \operatorname{ch}\|\phi\|_{2} \leq \operatorname{ch}|g|_{0} .
$$

Similarly, if we choose for $\hat{\boldsymbol{\beta}}_{h}$ the $V_{h}$-interpolate of $\boldsymbol{\beta}_{t}$, we get from (9)

$$
\left|\hat{\boldsymbol{\beta}}_{h}-\boldsymbol{\beta}_{t}\right|_{0}+h\left\|\hat{\boldsymbol{\beta}}_{h}-\boldsymbol{\beta}_{t}\right\|_{1} \leq c h^{2}\left\|\boldsymbol{\beta}_{t}\right\|_{2} \leq c h^{2}|g|_{0}
$$

If we choose for $\hat{p}_{h}$ the Clément interpolate of $p_{t}$ (see [4, problem 3.2.3]) we also deduce from (9) that

$$
\left\{\begin{array}{l}
\left|\hat{p}_{h}-p_{t}\right|_{0}+h\left|\hat{p}_{h}-p_{t}\right|_{1} \leq c h\left|p_{t}\right|_{1} \leq c h|g|_{0} \\
\left|\hat{p}_{h}-p_{t}\right|_{1} \leq c h\left|p_{t}\right|_{2} \leq c h t^{-1}|g|_{0} .
\end{array}\right.
$$

Finally, taking (25), (26) and (27) into account, we infer from (24) that

$$
\left\|\hat{\boldsymbol{\beta}}_{h}-\boldsymbol{\beta}_{h}\right\|_{1}+(t+\alpha h)\left|\hat{p}_{h}-p_{h}\right|_{1} \leq C h|g|_{0}
$$

with $C$ independent of $t$. Now, let $\hat{w}_{h}$ be the $W_{h}$-interpolate of $w_{t}$. Arguing as above, we get

$$
\begin{aligned}
\left\|\hat{w}_{h}-w_{h}\right\|_{1} & \leq C\left(h\left\|w_{t}\right\|_{2}+h\left\|\beta_{t}\right\|_{2}+t^{2} h\|\phi\|_{2}\right) \\
& \leq C h|g|_{0} .
\end{aligned}
$$

To conclude, one uses the interpolation error estimates (25)-(27), (28), (29) and the triangle inequality.

Remark 4. Concerning $\Gamma_{t}$, let $\Gamma_{h}=\left(\nabla \phi_{h}+\operatorname{rot} p_{h}\right) / \nu$. We have

$$
\begin{aligned}
\left|\Gamma_{t}-\Gamma_{h}\right|_{0} & \leq C\left(\left|\phi_{t}-\phi_{h}\right|_{1}^{2}+\left|p_{t}-p_{h}\right|_{1}^{2}\right)^{1 / 2} \\
& \leq C\left(h+\frac{h}{t+\alpha h}\right)|g|_{0} \leq c \alpha^{-1}|g|_{0},
\end{aligned}
$$

where $c$ is independent of $h$ and $t$. Thus, the $L^{2}$-error on $\Gamma_{t}$ is uniformly bounded in $t$.

As to the choice of $\alpha$, looking at (24), we see that, in (23), $c=O(\alpha)$ as $\alpha \rightarrow$ $\infty$, whereas $c=O\left(\alpha^{-1}\right)$ as $\alpha \rightarrow 0$. Thus, $\alpha$ should be taken moderately big. Unfortunately, the determination of the optimal value seems to be a tricky affair and will require some numerical experiments.

We conclude by saying that the above discretization can be used with other types of elements, in particular with the bilinear quadrangle to which the above proof can be applied mutatis mutandis. On the other hand, as observed in [3], because of 
the possible unboundedness of $\left|p_{t}\right|_{2}$, the use of higher-order elements is not clearly advantageous.

Département de Mathématiques

Université Laval

Québec G1K 7P4, Canada

1. K. J. BAThe, Finite Element Procedures in Engineering Analysis, Prentice-Hall, Englewood Cliffs, N. J., 1982.

2. K. J. BATHE \& F. BREZZI, "On the convergence of a four-node plate bending element based on Mindlin Reissner plate theory and a mixed interpolation," Mafelap V (J. Whiteman, ed.), Academic Press, 1985, pp. 491-503.

3. F. BREzzi \& M. Fortin, "Numerical approximation of Mindlin-Reissner plates," Math. Comp., v. 47, 1986, pp.151-158.

4. P. Ciarlet, The Finite Element Method for Elliptic Problems, North-Holland, Amsterdam, 1978.

5. P. Destuynder, Thèse d'état, Paris, 1980.

6. R. TEMAM, Navier-Stokes Equations, North-Holland, Amsterdam, 1977.

7. O. C. ZIENKIEWICZ, The Finite Element Method in Engineering Sciences, McGraw-Hill, London, 1978. 